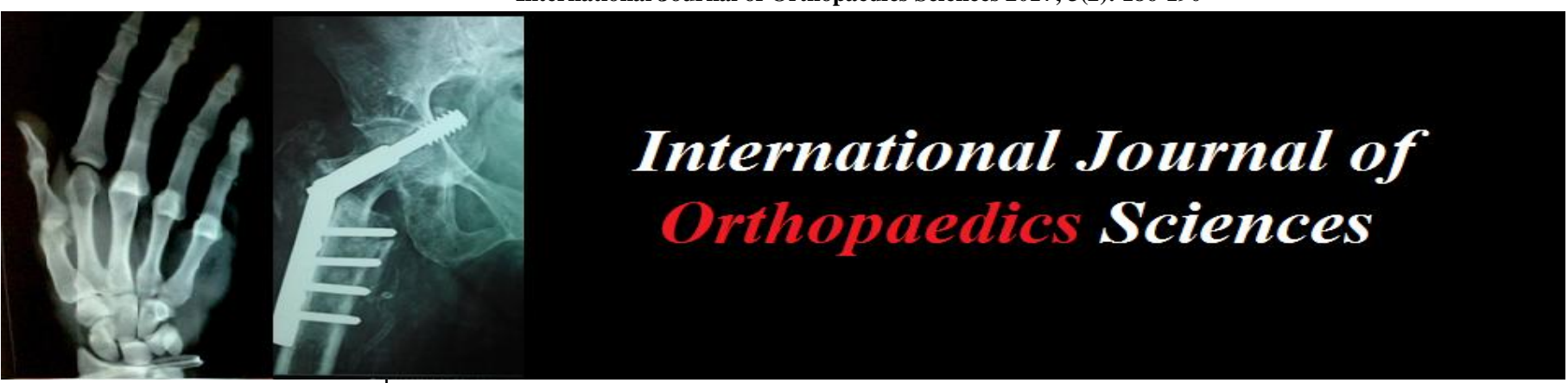

ISSN: $2395-1958$

IJOS 2017; 3(2): 186-190

(C) 2017 IJOS

www.orthopaper.com

Received: 29-02-2017

Accepted: 30-03-2017

Dr. Prateek Jain

MS Ortho, Deptt. of Orthopedics IGMC Shimla, India.

Dr. Ankur Gupta

MS Ortho, Deptt. of Orthopedics IGMC Shimla, India.

Dr. Ravikant Thakur

MS Ortho, Deptt. of Orthopedics

IGMC Shimla, India.

Dr. Shashikant Sharma

MS Ortho Deptt. of Orthopedics

IGMC Shimla, India.

\section{Stabilization of Distal Humerus fractures by precontoured bi-condylar plating in a $90-90$ pattern}

\author{
Dr. Prateek Jain, Dr. Ankur Gupta, Dr. Ravikant Thakur and Dr. \\ Shashikant Sharma
}

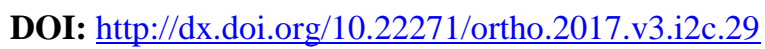

\section{Abstract}

Introduction:

The treatment of fractures of the distal humerus is difficult and involves the risk of bad functional results, particularly with articular fractures or impaired bone quality. Anatomical reconstruction and rigid fixation allowing early mobilization of the elbow are the basic prerequisites for good clinical outcomes. The aim of our study was to evaluate outcome of intercondylar fractures of distal humerus.

Patients and methods: The study included prospective or retrospective analysis of 28 cases of intercondylar fracture of distal humerus. There were $3 \mathrm{C} 1,15 \mathrm{C} 2$ and $10 \mathrm{C} 3$ type of fractures as per AO classification in our study. Patients were operated by 90-90 plating using olecranon osteotomy or Campbell's approach. Results were evaluated by post-operative X-rays, mayo elbow performance score and range of motion measurements.

Results: Male to female ratio in our study was $1.15: 1$. Mean age in study was $41.3 \pm 17.0$ yrs. Most common mode of injury was fall from height followed by road traffic accident. Mean duration of interval between injury and surgery was 7.1 \pm 7.3 days and mean duration of hospital stay was $10.3 \pm 6.1$ days. $85.72 \%$ of patients in had excellent to good results. Mean range of motion in our study was $100.6 \pm 20.0$ degrees. Complications included 1 case of transient ulnar nerve neuropathy, one case of deep infection, one case of malunion, and one case of delayed union.

Conclusions: Open reduction and internal fixation with $90-90$ plating provides reliable, rigid fixation allowing early functional mobilization of the elbow joint. Distal humeral locking plates proved to be useful in $\mathrm{C} 3$ type of fractures and in elderly osteoporotic bones. Complication rates in the study were low and comparable to studies in literature.

Keywords: Intercondylar fracture distal humerus, 90-90 plating.

\section{Introduction}

Intercondylar fractures of the distal humerus are uncommon injuries and present the most difficult challenge out of fractures of lower end of humerus. The complex shape of the elbow joint, the adjacent neurovascular structures, and the sparse soft tissue envelope combine to make these fractures difficult to treat ${ }^{[1]}$.

Improved surgical approaches and better fixation techniques allow early return to active motion, which decreases the rate of soft tissue complications. An understanding of distal humeral anatomy is helpful in the treatment of elbow fractures. In addition, biomechanical studies have helped define the optimal implant characteristics and placement ${ }^{[1]}$.

Distal humeral fractures have an estimated incidence in adults of 5.7 per 100,000 persons per year. They constitute $30 \%$ of humerus fractures and $2 \%$ of all the fractures. These injuries occur in a bimodal distribution, with an early peak in young males, twelve to nineteen years of age, and a second peak in elderly women, with osteoporotic bone ${ }^{[2,3]}$.

The majority of distal humerus fractures occur in one of two ways: low energy falls or high energy trauma. The most common cause is a simple fall in the forward direction. In general, $70 \%$ of patient that sustain an elbow fracture fall directly on to the elbow because they are unable to break their fall with an outstretched hand. High energy injuries are the cause of most distal humerus fractures in younger adults which include motor vehicle accidents, sports, fall from height, and industrial accidents ${ }^{[2,3]}$. The common signs and symptoms of distal humerus fractures are pain, swelling, deformity, and, sometimes, instability of the elbow after a fall. 
A complete neurovascular examination of the radial, median, ulnar, and anterior and posterior interosseous nerves should be completed both before and after any manipulation ${ }^{[4]}$.

AP and lateral radiographs of the elbow and humerus should be standard images. Traction radiographs can help define fragments that may be impacted or collapsed. CT scans with two dimensional views can be helpful. Three-dimensional reconstructions images help to know the exact geometry of fracture and often allow for subtraction of the radius and ulna. This technique can further illustrate fracture level, area and degree of comminution, articular incongruity, and column involvement. All of these factors can affect the surgical approach and ultimate treatment ${ }^{[4]}$.

Since the introduction of AO techniques open reduction and internal fixation has been the gold standard making conservative management almost obsolete. It is well accepted fact that plates are to be applied on both the columns in type $\mathrm{C}$ fractures, however, there is still no consensus regarding the orientation of plates on both the columns ${ }^{[5]}$.

The introduction of angular stable implants has revolutionized the operative treatment of these fractures, particularly in multifragmentory and osteopenia fractures. Now, anatomically precontoured plates with extensive distal screw options are available for distal humerus, promising enhanced stability and an ease in application ${ }^{[6]}$.

The aim of the present prospective-retrospective study was to evaluate the functional outcomes and complications in 28 patients of fracture intercondylar humerus operated in our institute using 90-90 plating.

\section{Patients and Methods}

The study included 28 cases of intercondylar fracture of distal humerus admitted in Indira Gandhi Medical College, Shimla, between June 2013 to November 2014 or those patients who had been operated before June 2013. In the retrospective group, records of patient were taken from record section. These patients were called for follow up, evaluated and assessed radiologically and functionally.

Upon arrival in the department of casualty, thorough clinical examination of patients were done including neurovascular examination. Radiography in form of X-rays and CT scans with 3D reconstruction were done to know the exact geometry of fracture. Patients were taken up for surgery after battery of blood investigations and detailed pre-anaesthetic examination. All the patients were operated in lateral position using either Campbell's approach or Chevron olecranon osteotomy approach, which was decided on preoperative planning and intraoperative visualization of fracture geometry. Ulnar nerve was identified and dissected on routinely basis. The intercondylar articular surface was visualized and the articular surface was reconstructed anatomically. The intraarticular reduction was stabilized with help of a $4.5 \mathrm{~mm}$ partially threaded cancellous screw passed over a guide wire. On attainment of a satisfactory articular reduction, the supracondylar ridges were examined and the shaft was reduced to the condyles and maintaining the medial and lateral ridges. The reduction was held with help of bone holding forceps and an interfragmentary screw was used to attain reduction if required. Precontoured distal humerus locking plates or reconstruction plates were applied over the lateral and medial ridges in a 90-90 fashion. Before definitive plate fixation, the elbow was placed through a range of motion to ensure there is no hardware impingement. The olecranon osteotomy was stabilized with tension band wiring principle. The elbow range of motion was again reassessed to rule out intraarticular impingement of the implant. Ulnar nerve was embedded in the soft tissue and a fat pad harvested locally was placed between the nerve and the medial plate if required. The triceps V-Y flap was reattached to the triceps muscle in case of the triceps turndown approach. We used 9090 plating using reconstruction plates or distal humerus precontoured plates as standard procedure.

Post operatively limb was immobilized in plaster of Paris slab for a period of 2 weeks. After 2 weeks sutures were removed and split age was discontinued and patients were put on physiotherapy.

Patients were followed up every 6 weeks for a period of 6 months. The results were assessed 6 months after the procedure using Rise borough and Radin grading system and Mayo elbow performance score system.

The collected data was tabulated on a computer using Windows 7 operating system on Microsoft office 2013, Excel package and the statistical analysis unpaired, two tailed Student's t-test and ANOVA (Analysis of variance) for qualitative variables was done on the same software and Epi Info ${ }^{\mathrm{TM}}$ version 7.

Chi square test, Fischer's exact test were used for univariate analysis of categorical variables in different subgroups. A pvalue $<0.05$ was considered statistically significant.

\section{Results}

There were 15 males and 13 females in our study. Mean age in our study was $41.35 \pm 17.05 \mathrm{yrs}$.

Majority of the patients (75\%) sustained injury due fall while $25 \%$ of the patients sustained injury due to road traffic accidents.

In our study maximum number of patients had 13C2 type of fracture of the distal humerus $(53.57 \%)$ followed by $13 \mathrm{C} 3$ type which constituted $35.71 \%$ of all the fractures. 13C1 type constituted only $10.71 \%$ of all the fractures. (Table 1 )

$60.71 \%$ of patients were operated by olecranon osteotomy approach, while $39.29 \%$ of patients were operated using Campbell's approach (triceps splitting). Olecranon osteotomy was more favourable in fractures with intraarticular comminution. However, relationship between fracture type and approach of surgery was statistically insignificant.

We observed comorbid conditions in $32.14 \%$ of our patients. $14.29 \%$ patients each had diabetes, $10.71 \%$ had epilepsy and $7.14 \%$ of patients had both diabetes and hypertension.

Maximum number of patients were operated within first week of injury. Delay occurred either due to delayed presentation to the institution or in the management of comorbid conditions which was statistically insignificant. Mean duration between injury and surgery was $7.71 \pm 7.38$ days and mean duration of hospital stay was $10.38 \pm 6.15$ days.

$42.86 \%$ patients had excellent results according to Mayo elbow performance score, $42.86 \%$ had good results, while $14.28 \%$ of the patients had fair and poor results.

$46.43 \%$ of the patients had range of motion of more than 100 degrees. $39.29 \%$ of the patients had range of motion more between $80-100$ degrees. Only $14.29 \%$ of the patients had range of motion less than 80 degrees.

Mean range of motion was $100.6 \pm 20.03$ degrees. (Table 1)

We observed that complexity of fracture increased with increasing age which was statistically significant with $p$ value of 0.03 . Patients with road traffic accidents had relatively complex fractures as compared with fall. However, this was statistically insignificant.

No statistical significant relationship was found while comparing various fracture subtypes with parameters like 
interval between injury and surgery, duration of hospital stay and post-operative complications.

Younger patients tend to have better range of motion, mayo elbow performance score and relatively less pain at final follow up as compared to older individuals. These observations were statistically insignificant.

No statistical significant relationship was found between interval between injury and surgery, duration of hospital stay, comorbid conditions and pain, range of motion and mayo elbow performance score at final follow up.

No statistical significant relationship was found between approach of surgery, post-operative complications and pain, range of motion and mayo elbow performance score at final follow up.

As the complexity of fractures increased, range of motion at final follow up decreased ( $\mathrm{p}$ value -0.0001 ).

Prolonged immobilization for a period of 4-6 weeks had relatively poorer outcome in terms of pain, range of motion, and mayo elbow performance score at final follow up.

Complication rate in our study was $14.28 \%$. There was 1 case of deep infection, 1 case of malunion, 1 case of delayed union, and 1 case of transient ulnar neuropathy.

Table 1: Data stratification according to AO classification

\begin{tabular}{|c|c|c|c|c|}
\hline \multicolumn{5}{|c|}{ AO Classification } \\
\hline & $\mathrm{C} 1$ & $\mathrm{C} 2$ & $\mathrm{C} 3$ & $P$ value \\
\hline No. of Patients & 3 & 15 & 10 & 0.43 \\
\hline Mean Age in years & $36.0 \pm 25.3$ & $35.0 \pm 14.6$ & $52.4 \pm 13.5$ & 0.03 \\
\hline \multicolumn{5}{|c|}{ Sex } \\
\hline Male & 2 & 10 & 3 & \multirow{2}{*}{0.17} \\
\hline Female & 1 & 5 & 7 & \\
\hline $\begin{array}{l}\text { Duration of hospital } \\
\text { stay }\end{array}$ & $9.6 \pm 1.1$ & $8.2 \pm 3.9$ & $13.6 \pm 8.8$ & 0.1 \\
\hline Range of Motion & $123.3 \pm 5.7$ & $108.0 \pm 12.1$ & $82.8 \pm 18.6$ & 0.0001 \\
\hline \multicolumn{5}{|c|}{ Mayo Elbow Performance Score } \\
\hline Excellent & 3 & 9 & 0 & \multirow{4}{*}{0.01} \\
\hline Good & 0 & 6 & 6 & \\
\hline Fair & 0 & 0 & 2 & \\
\hline Poor & 0 & 0 & 2 & \\
\hline
\end{tabular}

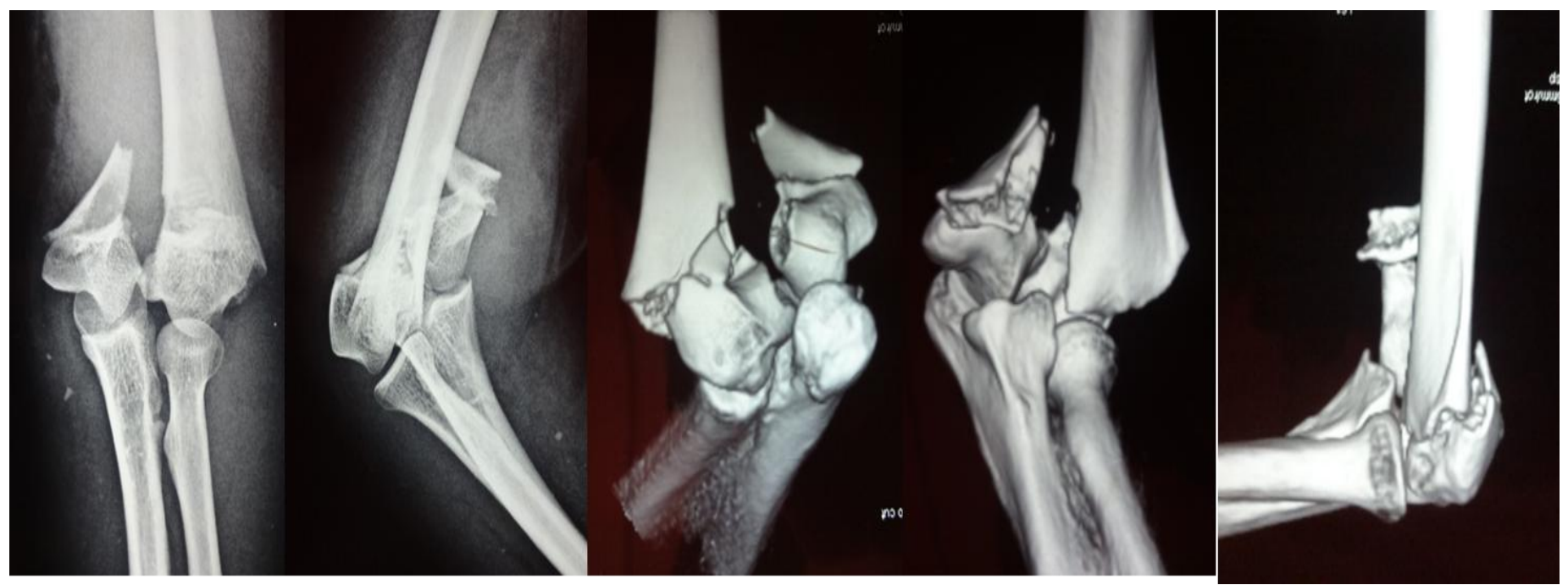

Fig 1: Pre-operative imaging

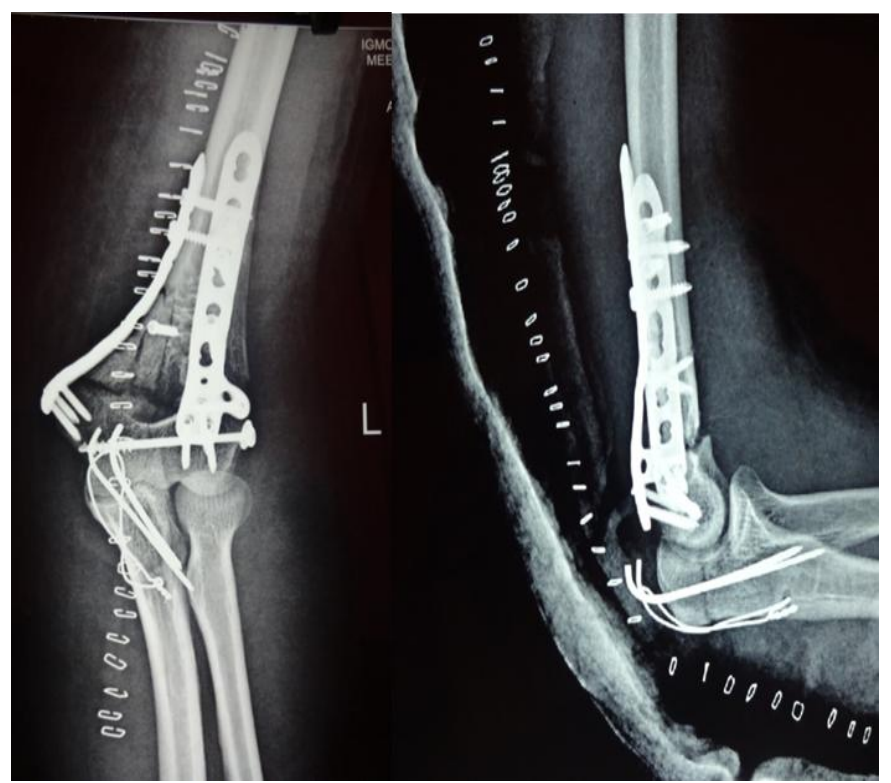

Fig 2: Post-operative $\mathrm{X}$ rays

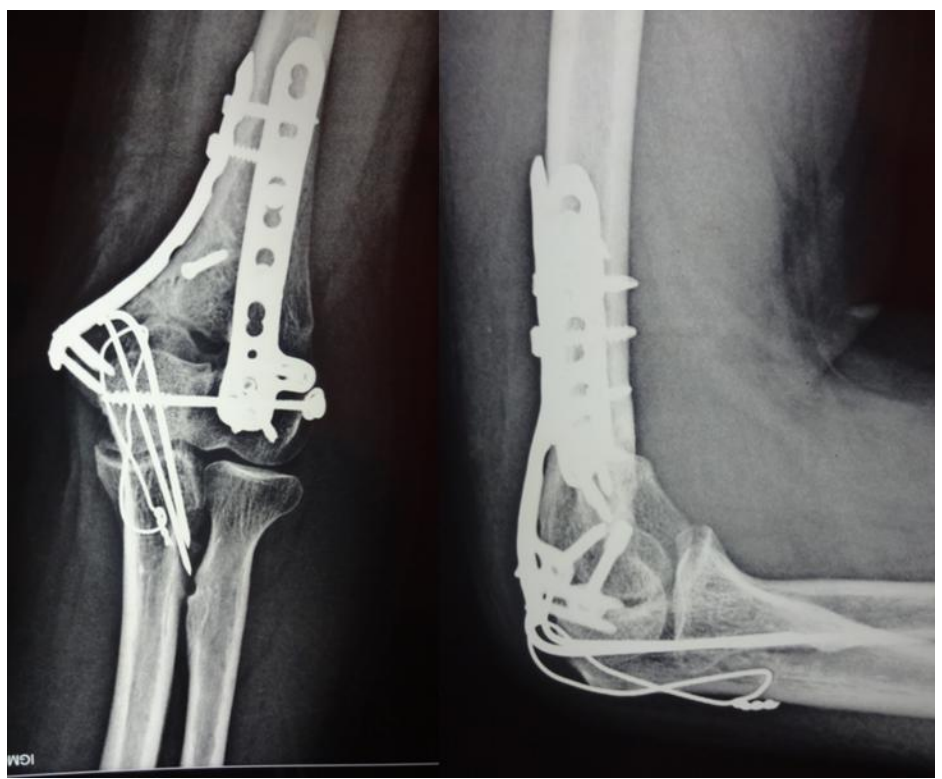

Fig 3.6: week follow up 


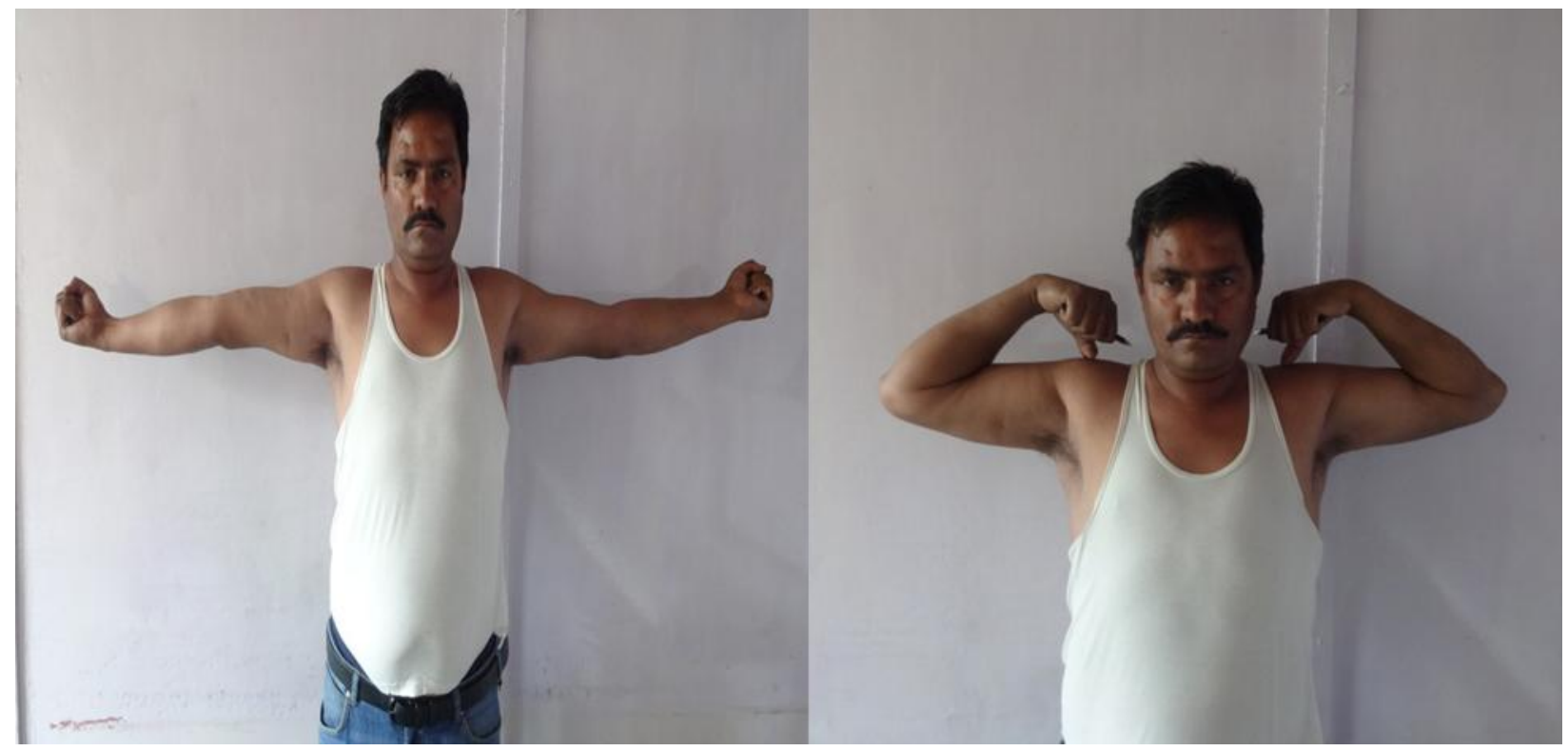

Fig 4.3: month follow-up with full range of motion

\section{Discussion}

24 out of 28 patients had excellent to good results in our study. There was tendency towards better results among younger individuals. However, we could not found statistically significant association.

There was tendency towards relatively complex fractures in older individuals, which may be on account of decreased bone quality and increased incidence of osteoporosis among older individuals. Patients with C3 type of fractures had relatively poorer outcome in terms of MEPS, ROM, and post-operative pain at final follow up, consistent with other studies ${ }^{[7,8]}$. These correlations were statistically significant.

The selection of a surgical approach for the management of distal humerus fractures is dependent on several factors. These include the surgeon's experience and preferences, fracture pattern, degree of articular involvement ${ }^{9}$. We used Campbell's approach in 11 patients and olecranon osteotomy in 17 patients. We felt that $\mathrm{C} 1$ and some $\mathrm{C} 2$ type of fractures (without much rotation of fragments) did not require olecranon osteotomy and could easily be operated by Campbell's approach. However, C2 type of fractures with significant rotational component and C3 type of fractures were better operated upon with olecranon osteotomy due to better visualization of articular surface as observed by Wilkinson et al. ${ }^{[10]}$.

The use of locking plates for distal humeral fractures remains controversial, and the indications for their use are unclear. The high cost of these implants requires that their use be justified by sufficient clinical evidence prior to their application. Two clinical case series on the results of locked plate fixation of distal humeral fractures have been reported by Greiner et al and Reising et al. ${ }^{[11,12]}$. Pooled analysis showed good/excellent results in $79 \%$ of the patients, with only a single case of implant failure.

Biomechanical studies have shown that locking plates provided somewhat improved fixation in models of osteoporotic or comminuted distal humeral fractures ${ }^{[13-15]}$. Despite the lack of available evidence in support of locking plates, many experts believe that their use may be advantageous in the management of comminuted, osteoporotic fractures.

We used reconstruction plates as well as distal humerus precontoured locking plates in the management of intercondylar fractures. We felt that fractures with larger distal fragments could be fixed with reconstruction plates, while fractures with smaller distal fragments or osteoporotic bone required precontoured locking plates due to greater distal screw options.

Another area of controversy is regarding the positioning of plates. In biomechanical studies, Korner et ${ }^{14}$ al found greater stability for perpendicular plate system while Stoffel et al. ${ }^{[13]}$ found greater rotational stability for parallel plating system while comparing parallel arrangement of mayo elbow system to perpendicular distal humerus plating system. We were able to achieve rigid fixation in all cases by using $4.5 \mathrm{~mm}$ partially threaded cancellous screw for intercondylar fragments in addition to perpendicular plating.

We observed that prolonged immobilization for a period of 46 weeks led to detrimental effect on post-operative pain, range of motion and mayo elbow performance score at final follow up which was statistically significant. These observations were similar to Korner et al. ${ }^{[8]}$ and Gupta et al. ${ }^{[7]}$.

We encountered only 1 case of ulnar neuropathy, which recovered completely. This may be due to routine intraoperative identification and protection of ulnar nerve. There was 1 case of deep infection which required debridement and removal of implant. Infection subsided and patient could return to his routine activities. 1 case of delayed union was treated with bone grafting at later stage and union could be achieved. There was 1 case of malunion in our study. Overall complication rates in our study were comparable to other studies ${ }^{[16-20]}$.

There were many limitations in our study. Sample size in our study was relatively small. Study was a prospective retrospective study. We used only orthogonal plating in our study. So comparison with other plating techniques like parallel or dorsal plating could not be done.

\section{Conclusions}

In the end, we conclude that from literature and from the experience of our study early open reduction and rigid internal fixation with dual plates in orthogonal fashion followed by early post-operative mobilization are to be recommended for the management of intercondylar fractures of the distal humerus. Precontored distal humeral locking plates is a good option for fractures with intraarticular 
comminution and smaller distal fragments as well as in older patients with osteoporotic bone. However, role of reconstruction plates in $\mathrm{C} 1$ and $\mathrm{C} 2$ type of fractures should not be underestimated. Controversy regarding positioning of plates still exists and needs larger prospective trials with longer duration of follow up.

\section{References}

1. Kuntz Jr, David G, Baratz ME. Fractures of the elbow. Orthop Clin North Am Jun. 1999; 30(1): 37-61.

2. George S. Athwal Fractures of the distal humerus. Chapter-33 in Rockwood and Green's fractures in adults. 7th Edn. Lippincott Williams and Wilkins. 945-998.

3. Robinson CM, Hill R, Jacobs N, Dall G, Brown C. Adult humeral metaphyseal fractures. Epidemiology and results of treatment. J Of Orthop Trauma. 2003; 17:38-47.

4. Galano GJ, Ahmed AS, Levine WN. Current treatment for bicolumnar distal humerus fractures. J Am Acad Orthop Surg. 2010; 18:20-30.

5. Nauth A, McKee MD, Ristevski B, Hall J, Schemitch EJ. Current concepts review. Distal humerus fractures in adults. J Bone Joint Surg (Am). 2011; 93:686-700.

6. Horlohe KHS, Bonk A, Wilde P, Becker L, Hoffmann R. Promising results after the treatment of smile and complex distal humerus type $\mathrm{C}$ fractures by angular stable double plate osteosynthesis. Orthop Traumatol Surg Res. 2013; 99:531-541.

7. Gupta R, Khanchandani P. Intercondylar fractures of the distal humerus in adults. A critical analysis of 55 cases. Injury. 2002; 33:511-515.

8. Korner J, Lill H, Muller LP et al. Distal humerus fractures in elderly patients. Results after open reduction and internal fixation. Osteoporos Int. 2005; 16:73-79.

9. Danny P Goel, Jeffery M Pike, George S Athwal. Operative techniques in orthopaedics. Oper Tech Orthop. 2010; 20:24-33.

10. Wilkinson JM, Stanley D: Posterior surgical approaches to the elbow: A comparative anatomic study. J Shoulder Elbow Surg. 2001; 10:380-382.

11. Reising K, Hauschild O, Strohm PC, Suedkamp NP. Stabilisation of articular fractures of the distal humerus: early experience with a novel perpendicular plate system. Injury. 2009; 40:611-7.

12. Greiner S, Haas NP, Bail HJ. Outcome after open reduction and angular stable internal fixation for supraintercondylar fractures of the distal humerus: preliminary results with the LCP distal humerus system. Arch Orthop Trauma Surg. 2008; 128:723-9.

13. Stoffel K, Cunneen S, Morgan R, Nicholls R, Stachowiak G. Comparative stability of perpendicular versus parallel double-locking plating systems in osteoporotic comminuted distal humerus fractures. J Orthop Res. 2008; 26:778-84.

14. Korner J, Diederichs G, Arzdorf M, Lill H, Josten C, Schneider E, Linke B. A biomechanical evaluation of methods of distal humerus fracture fixation using locking compression plates versus conventional reconstruction plates. J Orthop Trauma. 2004; 18:286-93.

15. Schuster I, Korner J, Arzdorf M, Schwieger K, Diederichs G, Linke B. Mechanical comparison in cadaver specimens of three different 90-degree doubleplate osteosyntheses for simulated C2-type distal humerus fractures with varying bone densities. J Orthop Trauma. 2008; 22:113-20.

16. Gofton WT, MacDermaid JC, Patterson SD, Faber KJ,
King GJW. Functional outcome of AO type C distal humeral fractures. J Hand Surg. 2003; 28:294-308.

17. Huang T, Chiu F, Chuang T, Chiu T. Surgical treatment of acute displaced fractures of adult distal humerus with reconstruction plate. Injury. 2004; 35:1143-1148.

18. Liu J, Ruan H, Wang J, Fan C, Zang B. Double column fixation for type $\mathrm{C}$ fractures of the distal humerus in elderly. J Shoulder Elbow Surg. 2009; 18:646-651.

19. Li S, Li Z, Cai Z, et al. Bilateral plate fixation of type C distal humerus fractures. Experience at a single institute. Int Orthop. 2011; 35: 433-438.

20. Mardanpour K, Rahbar M. Open reduction and internal fixation of intraarticular fractures of humerus. Evaluation of 33 cases. Trauma Mon. 2013; 17(4):396-400.e 